\title{
An array of surface-enhanced Raman scattering substrates based on plasmonic lenses
}

\author{
Mehmet Kahraman ${ }^{1}$, Semih Cakmakyapan ${ }^{2}$, Ekmel Ozbay $^{2,3}$, and Mustafa Culha, ${ }^{1, *}$ \\ Received 7 June 2012, revised 30 August 2012, accepted 7 September 2012 \\ Published online 1 October 2012
}

An array of ring-shaped holes is prepared from silver thin films using electron beam lithography. The optimal conditions for high performance as a surface-enhanced Raman scattering (SERS) substrate are investigated. Either the diameter of the hole $(0.5,1.0,2.0,3.0$ and $4.0 \mu \mathrm{m})$ or the slit width $(200,300,400,500$ and $600 \mathrm{~nm})$ is varied. 4-Aminothiophenol (ATP) adsorbed on the structures as a self-assembled monolayer (SAM) is used as probe to evaluate the SERS performance of the generated structures. It is found that there is an optimal configuration for ringshaped holes with a 3.0- $\mu$ m diameter and 200-nm slit width. The SERS activity on this optimal lens configuration is found to be 13 times greater than that of the activity on the silver thin film. An array of these structures at this optimal configuration can easily be constructed and used in a range of SERS-based sensing applications.

\section{Introduction}

Surface plasmons are collective oscillations of conduction-band electrons on a noble-metal surface excited by electromagnetic light. The interaction of light and noblemetal surfaces is known as "plasmonics", which is a growing field of research and an emerging branch of nanophotonics. The nanophotonics research mainly focuses on understanding and manipulation of surface plasmons generated on metal surfaces. The field has attracted great attention due to the potential application of optical devices by controlling, manipulating and amplifying light on the nanometer scale, constructing novel sensors, and building photonics circuits [1-8]. Furthermore, biomedical applications of plasmonic structures such as medical diagnostics and therapeutics are a rapidly growing research area [9-16].

The investigation of use of optical properties (scattering and absorption) of noble-metal nanostructures has been pursued by several groups [17-22]. The study of interactions of molecules or molecular structures with plasmonic nanostructures is another rapidly growing research area having significant impact on other applications such as surface-enhanced Raman spectroscopy, nanoscale optical spectroscopy and surface plasmon resonance spectroscopy [23-27]. Depending on the noblemetal structure used, the surface plasmons can be classified into two groups. The SPPs occur on smooth thin films with thicknesses in the range of 10-200 nm of noble metals such as silver and gold. The localized surface plasmons (LSPR) are excited on isolated nanostructures such as nanoparticles or lithographically prepared nanostructures [27-29]. The properties of surface plasmons depend on the thickness of metal film, type of metal and roughness of the metal surfaces and dielectric constant of the adjacent medium. However, the LSPR occur on silver and gold nanoparticles in the range of $10-200 \mathrm{~nm}$ particle sizes and its properties mostly depend on size, type, and shape of nanoparticles [30-33].

When a Raman-active molecule is subject to intensified electromagnetic fields (surface plasmons), the magnitude of the induced dipole moment increases. Thus, the intensity of Raman scattering obtained from the active molecule increases [34]. This phenomenon is known as surface-enhanced Raman scattering (SERS) and has emerged as a powerful technique used for detection, identification and characterization of biological mole-

\footnotetext{
* Corresponding author E-mail: mculha@yeditepe.edu.tr or mculha2@gmail.com, Phone: +90-216-5781587, Fax: +90-2165780829

1 Department of Genetics and Bioengineering, Faculty of Engineering and Architecture, Yeditepe University, Atasehir, Istanbul, Turkey

2 Department of Physics and Nanotechnology Research Center, Bilkent University, 06800 Ankara, Turkey

3 Department of Electrical and Electronics Engineering, Bilkent University, 06800 Ankara, Turkey
} 
cules and structures such as proteins [35, 36], DNA and RNA [37, 38], bacteria [39, 40], yeast [41], and living cells $[42,43]$, and a variety of nonbiological molecules $[44,45]$. The two separate processes are considered to contribute to the enhancement mechanism; electromagnetic and chemical enhancement [46-49]. Electromagnetic enhancement is strongly related to surface plasmons (SPs) of noble-metal nanostructures and considered as the principal component of the enhancement mechanism. However, the contribution of chemical enhancement is rather limited (up to two orders of magnitude) and its magnitude depends on the chemical structure of the molecule $[34,50]$. Since the electromagnetic enhancement through the surface plasmons is the major contributing to the SERS enhancement mechanism, the construction of plasmonic structures for the optimization and manipulation of the surface plasmons is the focal point for the preparation of optimally performing SERS substrates $[29,51,52]$. Substrate effects on SERS enhancement for the case of milled metal nanoparticles were investigated by Rosa et al. [53]. The electromagnetic mechanism of SERS predicts an SERS enhancement factor, which is determined by the fourth power of the field $\left(|E|^{4}\right)$ enhancement in the local optical fields of metal surfaces $[29,50,54]$.

An incident electromagnetic field can be enhanced and localized in the slit region of a metal film [55]. This localized field couples surface plasmons on the surface of the metal. Surface plasmon polaritons propagate along the metal surface. The propagation length of surface plasmon polaritons can be expressed by [56]:

$d_{\mathrm{SPP}}=\lambda_{0} \frac{\left(\varepsilon_{m}^{\prime}\right)^{2}}{2 \pi \varepsilon_{m}^{\prime \prime}}\left(\frac{\varepsilon_{m}^{\prime}+\varepsilon_{d}}{\varepsilon_{m}^{\prime} \varepsilon_{d}}\right)^{3 / 2}$,

where $\lambda_{0}$ is wavelength of free space, $\varepsilon_{m}^{\prime}$ and $\varepsilon_{m}^{\prime \prime}$, are real and imaginary parts of relative permittivity, respectively, and $\varepsilon_{d}$ is the permittivity of the dielectric medium. This formula suggests which metal is favorable in terms of propagation length at a given frequency. For the case of a circular slit structure, propagating surface plasmon polaritons focus at the center of the circle and create standing waves, thus they can operate like a plasmonic lens [57]. It was also shown that field enhancement and focusing of surface plasmon polaritons at the center is possible by circular slits into a silver film [58]. The dependence of the circle diameter on the enhancement at the center was reported to increase almost linearly with radius $r$ for the cases where the propagation length of surface plasmon polaritons is much smaller than the radius of the ring [57].

In this study, circular plasmonic lens with different ring diameter and slit width were prepared by EBL and the influence of plasmonic lens structure on SERS enhancement was investigated using self-assembled p-ATP molecules on the plasmonic lenses. The SERS enhancement performance of the lenses was evaluated by comparing the enhancement between planar silver thin film and circular plasmonic lenses. The optimal configuration is easy to prepare and use as a SERS substrate in variety of sensing applications.

\section{Experimental}

\subsection{Preparation of circular plasmonic lenses}

Circular slit structures were fabricated by using electron beam lithography. First, samples were spin coated by positive-tone electron beam resist. Then, a circular area, excluding the slit region, was exposed on a Raith electron beam lithography system. 100-nm thick silver was deposited on the samples by electron beam evaporation. After the standard lift-off process, ring-shaped holes with different width and radii were obtained, as shown in Fig. 1 . The results obtained from the unpatterned part of the metal in order to calculate the SERS enhancement were compared to those obtained from the slit structures. Various rings have been fabricated with different inner and outer radii. Table 1 shows the details of the fabricated lens arrays. The plasmonic lens arrays were characterized using a Karl Zeiss EVO 40 model scanning electron microscopy (SEM) instrument.

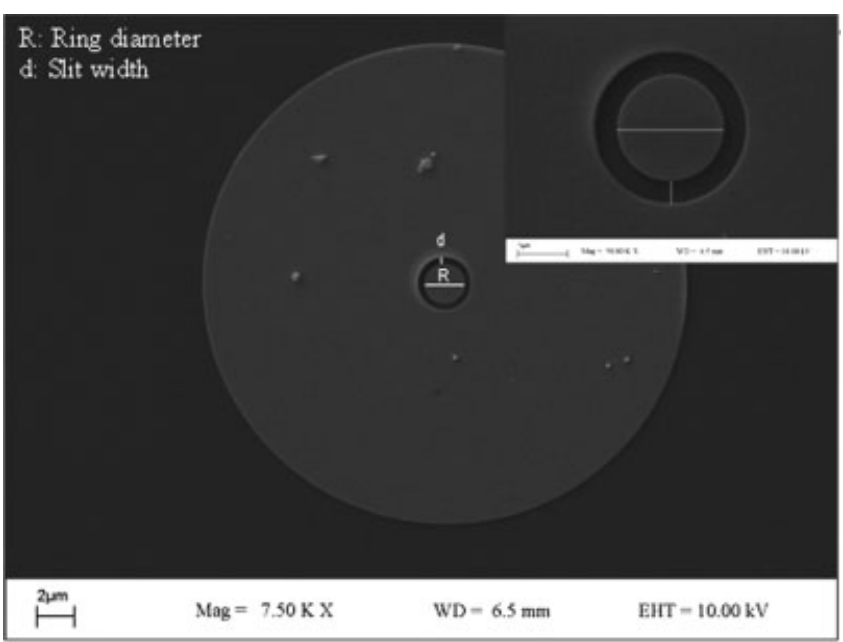

Figure 1 SEM image of ring-shaped hole on silver film. 

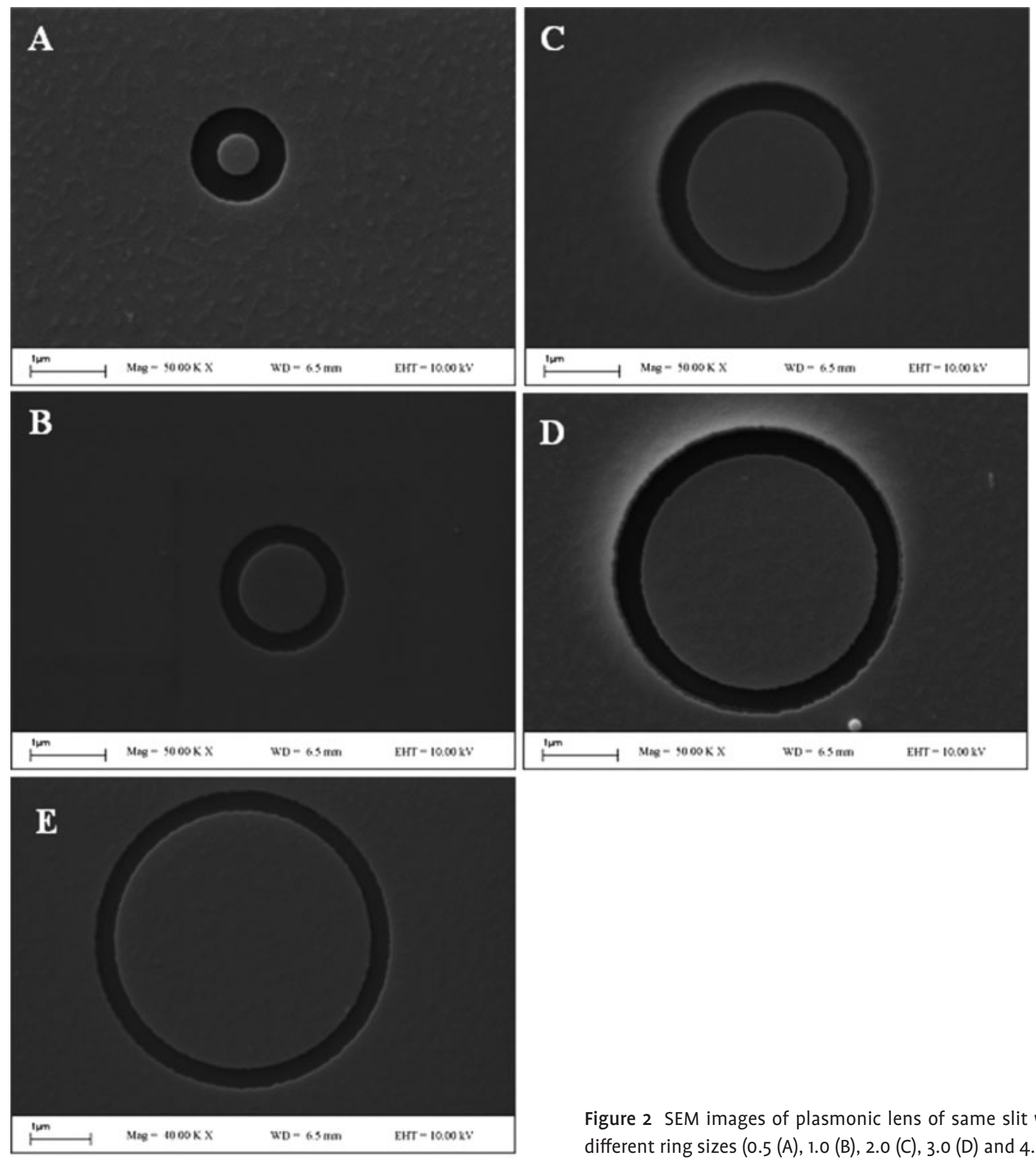

Figure 2 SEM images of plasmonic lens of same slit width with different ring sizes (0.5 (A), 1.0 (B), 2.0 (C), 3.0 (D) and $4.0 \mu \mathrm{m}$ (E).

Table 1 Plasmonic lenses with different ring diameters and slit widths used in the study.

\begin{tabular}{|l|l|l|l|l|l|}
\hline Sample number & $\mathbf{1}$ & $\mathbf{2}$ & $\mathbf{3}$ & $\mathbf{4}$ & $\mathbf{5}$ \\
\hline Ring diameter $(\mu \mathrm{m})$ & 0.5 & 1.0 & 2.0 & 3.0 & 4.0 \\
Slit width $(\mathrm{nm})$ & 200 & 300 & 400 & 500 & 600 \\
\hline
\end{tabular}

\subsection{Self-assembly of Raman-active molecule on plasmonic lens arrays}

4-aminothiophenol (p-ATP) is used to study the SERS enhancement property of the prepared lenses. Since this molecule has a free thiol (-SH) group, it forms a quite uniform monolayer, which is called a self-assembled monolayer (SAMs), on the noble-metal surfaces [59]. There- 
fore, the prepared plasmonic lens chips were immersed in an ethanolic solution of 4-aminothiophenol (p-ATP) $(1 \mathrm{mM})$ for $2 \mathrm{~h}$ to form SAM. Then, the surface was washed with ethanol to remove the nonspecifically adsorbed molecules from the surface.

\subsection{Raman instrumentation}

All measurements were performed using a completely automated Renishaw InVia Reflex Raman microscopy system equipped with an 830-nm diode and 514-nm argon-ion lasers. Laser with a wavelength of $514 \mathrm{~nm}$ was used to excite the surface plasmons on the plasmonic lenses prepared as the silver thin film with e-beam deposition. The laser power was $25 \mathrm{~mW}$ for all experiments unless stated otherwise. A 50 $\times$ microscope objective (NA = 0.75 ) was used to focus the laser beam to obtain the SERS spectra from the self-assembled ATP molecules on the plasmonic lenses.

\section{Results and discussion}

\subsection{Characterization of plasmonic lenses}

The silver plasmonic lenses with different diameters and slit widths on a $25-\mu \mathrm{m}$ circular silver thin film with a $100 \mathrm{~nm}$ thickness were prepared and characterized with SEM as seen in Fig. 1. Figure 2 shows the SEM images of plasmonic lenses having the same slit width $(400 \mathrm{~nm})$ but different inner ring diameters $(0.5,1.0,2.0,3.0$, and $4.0 \mu \mathrm{m})$. When the SEM images were examined, the ring diameters were about ten percent larger and the slit width of plasmonic lenses was about ten percent smaller than the theoretically targeted structures.

\subsection{Influence of plasmonic lens structure on SERS enhancement}

The electromagnetic enhancement component whose relationship with the surface plasmons is now well established has a major role in SERS enhancement. The enhancement factor in SERS experiment is the fourth power of the electromagnetic field of surface plasmons generated on nanostructure surfaces $[29,50,54]$. This means that the higher the intensity of surface plasmons, the greater the SERS enhancement. Plasmonic lenses have the capability of focusing the surface plasmon depending on their structural properties such as shape and size $[57,58,60,61]$. Although near-field optical microscopy (NSOM) has generally been used for the optical characterization of plasmonic lenses, we have used SERS for the optical characterization and evaluation of plasmonic lenses for their performance as SERS substrate. p-ATP as a Raman-active molecule was self-assembled on silver plasmonic lenses to compare the SERS performance of them depending on their ring diameters and slit widths. An average of ten SERS spectra obtained from each lenses was used for the comparison.

The spectral reproducibility from lens to lens was first investigated by collecting one spectrum from each lens on the ten-lens array. Figure 3 shows the SERS spectra obtained from the array composed of a $2.0-\mu \mathrm{m}$ ring diameter and 150-nm slit width. As seen, the reproducibility of SERS spectra is satisfactory for comparison purposes.

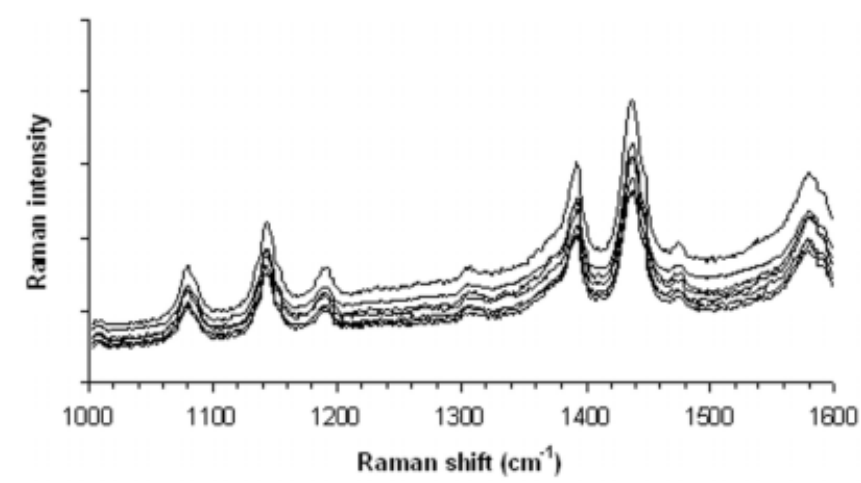

Figure 3 Ten SERS spectra obtained from the equivalent plasmonic lenses on the same array.

The number of the Raman-active molecules under the microscope objective is assumed to be the same since p-ATP forms a SAM on the silver surfaces. The SERS performance of the constructed lenses was found to be lens diameter and slit width dependent. The SERS spectra obtained from the plasmonic lens with $0.5-\mu \mathrm{m}$ diameter and 200-nm slit width and regular planar silver film, which was a part of the array system, was compared. When the most intense peak at $1435 \mathrm{~cm}^{-1}$ on the SERS spectra was chosen to make the comparison, it was found that the intensity of this peak was about 2 times higher than the peak on the SERS spectra obtained on the regular silver thin film. As an example, Fig. 4 also demonstrates the SERS spectra obtained from plasmonic lenses with 2- $\mu \mathrm{m}$ diameter and 200-nm slit width (a) and a regular thin film (b). The intensity of the chosen peak is about 4 times higher on the plasmonic lens than that of the silver thin film. This means that when the ring diameter increases, the intensity of SERS spectra increases. 


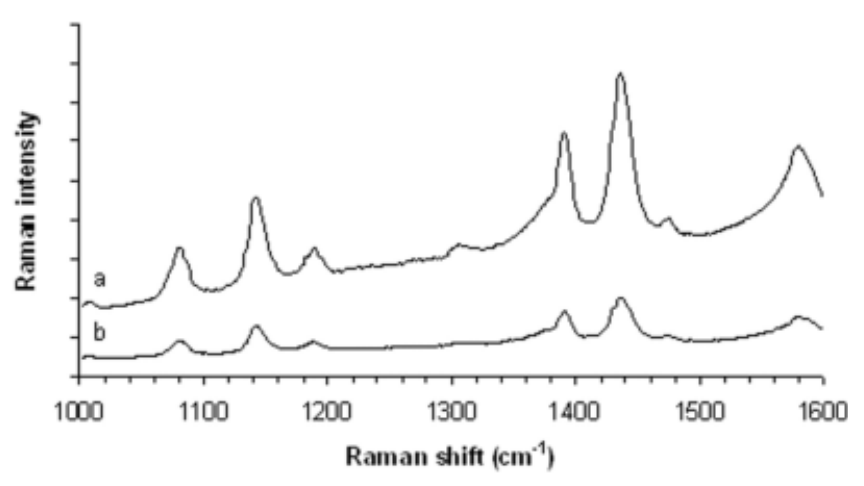

Figure 4 SERS spectra of p-ATP obtained from a plasmonic lens (a) and a silver thin film (b).

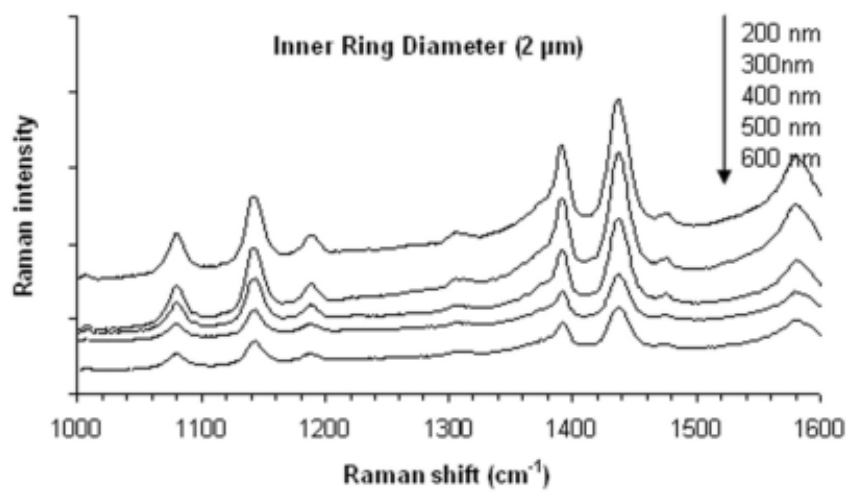

Figure 5 SERS spectra obtained from plasmonics lenses with different slit widths.

The influence of slit width on SERS activity was investigated next. While the diameter of ring-shaped holes is kept constant at $2 \mu \mathrm{m}$, their slit widths are varied. Figure 5 shows the SERS spectra obtained on the structures with increasing slit width. It is clear that as the slit width increases, the intensity of SERS spectra decreases. The same results were observed from the other plasmonic lenses having different ring diameters prepared in the study (data is not shown).

Figure 6 shows the graph of the $I_{\text {lens }} / I_{\text {film }}$ ratio for the lens with $3-\mu \mathrm{m}$ diameter with increasing slit width as an example. As is seen, the ratio of the intensity decreases with plasmonic lens having larger slit width. All ring-shaped lenses with different diameters show that the $200-\mathrm{nm}$ slit widths is the optimal. The intensity at the center $I$ of the ring is expressed with the following formula [57]:

$I=C I_{0} \frac{2 R}{\lambda_{\mathrm{SP}}} \exp \left(\frac{-R}{2 \mathrm{~d}_{\mathrm{SPP}}}\right)$.
In this expression, $C$ is the coupling coefficient, $I_{0}$ is the intensity of the incident beam, and $R$ is the radius of the inner circle. $\lambda_{\mathrm{SP}}$ is the surface plasmon polaritons wavelength, which is excited on the surface of the metal. The coupling coefficient $C$ is a critical parameter, which is related to the slit width. It was shown by Mehfuz et al. that the coupling coefficient $C$ decreases as slit width increases [62]. As a result, when the ring diameter is kept constant, the intensity at the center decreases as the slit width increases. Consequently, the results shown in Fig. 6 coincide with this theoretical approximation.

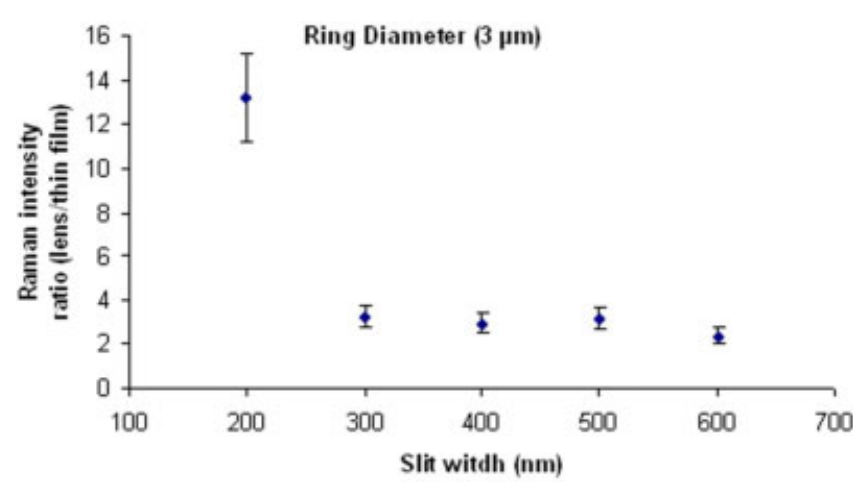

Figure 6 SERS intensity ratio depending on slit-width changes.

Next, the influence of the inner-ring diameter on SERS was investigated using plasmonic lenses with a 200$\mathrm{nm}$ slit width and increasing ring diameters. The SERS spectra obtained from the plasmonic lenses having ring diameters of 0.5, 1.0, 2.0, 3.0, 4.0 $\mu \mathrm{m}$ are shown in Fig. 7. Figure 8 shows the change of $I_{\text {lens }} / I_{\text {film }}$ ratio with the increasing diameter. The SERS intensity obtained from the plasmonic lens is 13.18 times higher compared to a planar silver thin film.

Figure 9 illustrates the FDTD simulation result for the case, where the diameter of the ring is $3 \mu \mathrm{m}$ and the slit width is $200 \mathrm{~nm}$. The electric field is localized inside the slit region. The reason for this localization is that the electromagnetic wave is enhanced due to the slits, which are smaller than the wavelength of the incident light [63]. The simulations were carried out by using TM-polarized Gaussian beam, where the electric-field component of the electromagnetic wave is in the $X$-direction. Surface plasmon polaritons are excited normal to the slits. Therefore, the electric-field intensity becomes higher in the slit region along the $Y=0$ line, which is supported by the simulation in Fig. 9. The electric-field distribution on the surface of the structure was monitored. It is also seen that the field is focused in the center of the circle. The 


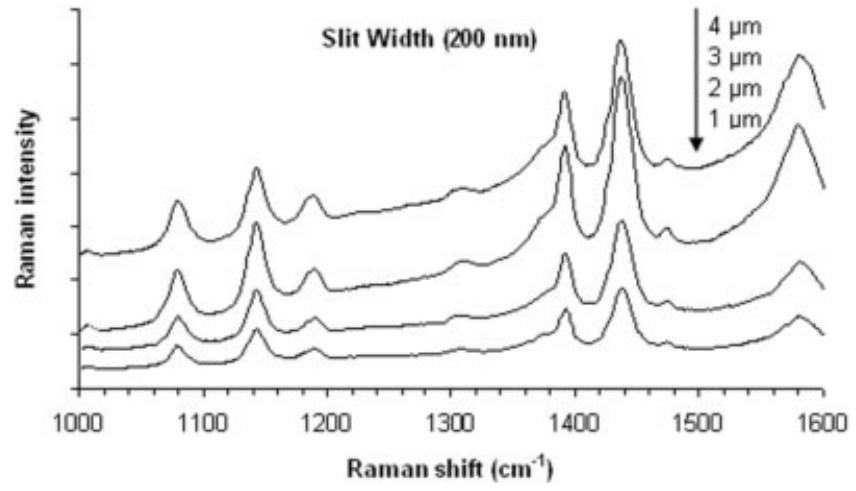

Figure 7 SERS spectra obtained from plasmonic lenses having the same slit width with different ring diameters.

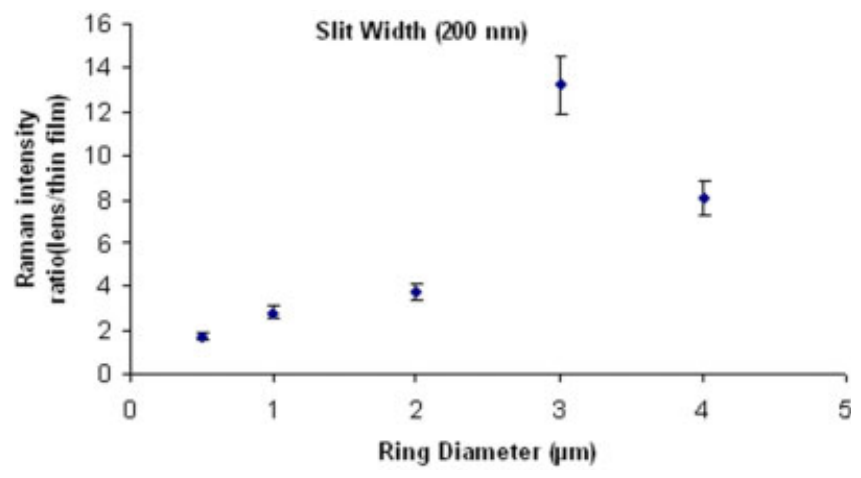

Figure 8 SERS intensity ratio depending on ring-diameter changes.

propagation loss is proportional to $\exp \left[-\operatorname{Im}\left(k_{\mathrm{SP}}\right) r\right]$, as the surface plasmons polaritons propagate towards the center of the ring [58]. Therefore, it is observed that the field intensity at the slit region is higher than the center of the rings. We integrated the square of the electricfield intensity on the surface for both patterned and unpatterned metal, and took the ratio of the integrations for the comparison. The intensity of electric-field ratio $\left(\left|E_{\text {lens }}\right|^{4} /\left|E_{\text {film }}\right|^{4}\right)$ was calculated and found to be 12.9 .

The FDTD simulation results also demonstrate that when the inner ring diameter is increased with a constant slit width, the electric-field intensity on plasmonic lenses increases. As the diameter of the ring increases, the area of the slit region increases, as well. This provides focusing of the excited surface plasmon polaritons towards the center more intensively. The results can also be explained by Eq. (2). In this case, $C$ is constant, since the slit width is kept constant. The intensity at the center should increase monotonically with $r$ up to surface plasmon propagation length $d_{\mathrm{SPP}}$ (Eq. (1)). The theoretical calculation is consistent with the experimental result

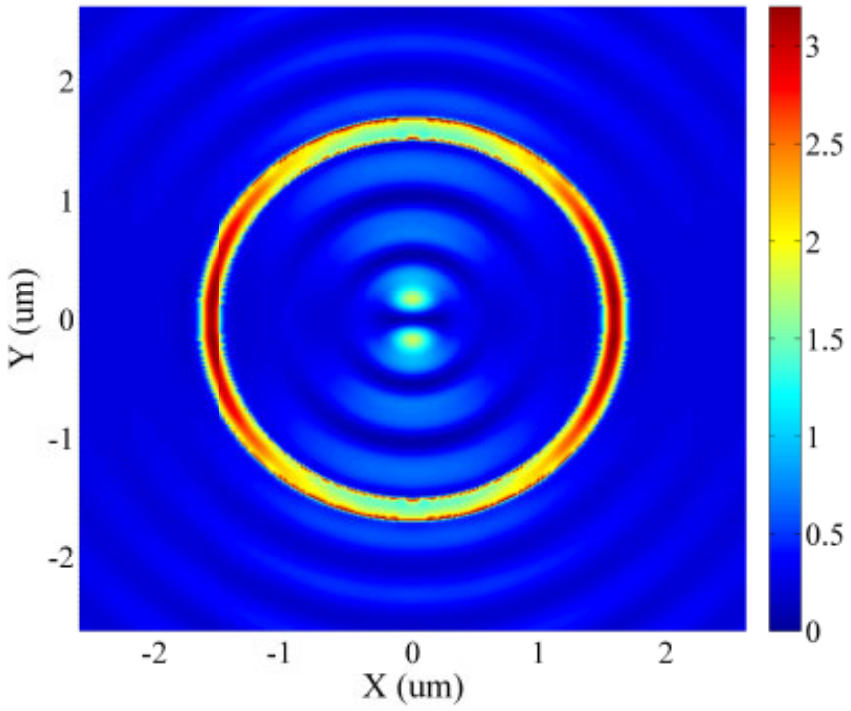

Figure 9 (online color at: www.ann-phys.org) Electric-field intensity $|E|^{2}$ distribution on the plasmonic lens.

and the literature [57] up to $4.0-\mu \mathrm{m}$ inner diameter. This is due to the size of the laser spot used for the SERS experiment. The electromagnetic radiation is necessary for the excitation of surface plasmons on the nanostructured metal. When the $50 \times$ objective is used, the laser spot size is much smaller than $4.0 \mu \mathrm{m}$ and cannot excite plasmons in the large area. Thus, the ratio of the electric-field intensity decreases.

\section{Conclusion}

The results show that when the slit width is increased, the SERS performance of plasmonic lenses decreases. The maximum SERS performance was obtained with the plasmonic lens having a 200-nm slit width for all plasmonic lenses with different ring diameters. The slit width was kept constant at $200 \mathrm{~nm}$ to investigate the influence of ring diameter on SERS. The SERS intensity obtained from plasmonic lens having $3.0-\mu \mathrm{m}$ inner diameter is 13.18 times higher compared to a planar silver thin film, which is consistent with the theoretical calculations. Since the preparation of the optimal configuration is very easy and can be done in an array format, we believe these structures have a pronounced potential as a SERS substrate for routine high-throughput chemo- and biosensing applications. 
Acknowledgements. The authors greatly acknowledge the financial support of Yeditepe University, Bilkent University and TUBITAK during the course of this study.

Key words. Ring-shaped holes, plasmon focusing, SERS, substrate.

\section{References}

[1] M. E. Stewart et al., Chem. Rev. 108, 494-521 (2008).

[2] H. A. Atwater, Sci. Am. 296, 56-63 (2007).

[3] E. Ozbay, Science 311, 189-193 (2006).

[4] S. A. Maier and H. A. Atwater, J. Appl. Phys. 98, 011101 (2005).

[5] R. P. Van Duyne, Science 306, 985-986 (2004).

[6] C. L. Haynes, J. Phys. Chem. B 107, 7337-7342 (2003).

[7] S. A. Maier et al., Nature Mater. 2, 229-232 (2003).

[8] H. J. Lezec et al., Science 297, 820-822 (2002).

[9] I. H. El-Sayed, X. Huang, and M. A. El-Sayed, Nano Lett. 5, 829-834 (2005).

[10] A. P. Alivisatos, Nature Biotechnol. 22, 47-52 (2004).

[11] N. L. Rosi and C. A. Mirkin, Chem. Rev. 105, 15471562 (2005).

[12] L. R. Hirsch et al., Proc. Natl. Acad. Sci. USA 100, 13549-13554 (2003).

[13] X. Huang, I. H. El-Sayed, W. Qian, and M. A. El-Sayed, J. Am. Chem. Soc. 128, 2115-2120 (2006).

[14] I. H. El-Sayed, X. Huang, and M. A. El-Sayed, Cancer Lett. 239, 129-135 (2006).

[15] D. P. O’Neal et al., Cancer Lett. 209, 171-176 (2004).

[16] C. A. Loo et al., Nano Lett. 5, 709-711 (2005).

[17] R. Elghanian et al., Science 277, 1078-1081 (1997).

[18] X. Huang, P. K. Jain, I. H. El-Sayed, and M. A. El-Sayed, Nanomedicine 2, 681-693 (2007).

[19] P. K. Jain, X. Huang, I. H. El-Sayed, and M. A. El-Sayed, Acc. Chem. Res. 41, 1578-1586 (2008).

[20] H. Li and L. Rothberg, Proc. Natl. Acad. Sci. USA 101, 14036-14039 (2004).

[21] D. C. Hone, A. H. Haines, and D. A. Russell, Langmuir 19, 7141-7144 (2003).

[22] C. L. Schofield, A. H. Haines, R. A. Field, and D. A. Russell, Langmuir 22, 6707-6711 (2006).

[23] B. Pettinger et al., Phys. Rev. Lett. 92, 096101 (2004).

[24] G. C. Schatz and R. P. Van Duyne, (eds.) Handbook of Vibrational Spectroscopy, Vol. 1 (Wiley, New York, MA, 2002), p. 759.

[25] A. J. Haes and R. P. Van Duyne, Anal. Bioanal. Chem. 379, 920-930 (2004).

[26] J. M. Brockman, B. P. Nelson, and R. M. Corn, Annu. Rev. Phys. Chem. 51, 41-63 (2000).

[27] K. A. Willets and R. P. Van Duyne, Annu. Rev. Phys. Chem. 58, 267-297 (2007).

[28] X. Lu et al., Annu. Rev. Phys. Chem. 60, 167-192 (2009).

[29] A. J. Haes et al., MRS Bull. 30, 368-375 (2005).
[30] J. Henzie et al., Annu. Rev. Phys. Chem. 60, 147-65 (2009).

[31] P. K. Jain, K. S. Lee, I. H. El-Sayed, and M. A. El-Sayed, J. Phys. Chem. B 110, 7238-7248 (2006).

[32] K. L. Kelly, E. Coronado, L. L. Zhao, and G. C. Schatz, J. Phys. Chem. B 107, 668-677 (2003).

[33] E. Hutter and J. H. Fendler, Adv. Mater. 16, 1685-1706 (2004).

[34] C. Haynes, A. D. Mcfarland, and R. P. Van Duyne, Anal. Chem. 340, 339-346 (2005).

[35] M. Kahraman, I. Sur, and M. Culha, Anal. Chem. 82, 7596-7602 (2010).

[36] S. Keskin, M. Kahraman, and M. Culha, Chem. Commun. 47, 3424-3426 (2011).

[37] L. Fabris et al., J. Am. Chem. Soc. 129, 6086-6087 (2007).

[38] Y. W. C. Cao, R. Jin, and C. A. Mirkin, Science 297, 1536-1540 (2002).

[39] M. Culha et al., Appl. Spectrosc. 62, 1226-1232 (2008).

[40] D. Cam et al., J. Raman Spectrosc. 41, 484-489 (2010).

[41] I. Sayin et al., Appl. Spectrosc. 63, 1276-1282 (2009).

[42] J. Kneipp et al., Nano Lett. 6, 2225-2231 (2006).

[43] S. Lee et al., Anal. Chem. 79, 916-922 (2006).

[44] M. Wang et al., J. Phys. Chem. C 111, 3038-3043 (2007).

[45] O. Cozar et al., J. Mol. Struct. 788, 1-6 (2006).

[46] M. Moskovits, J. Chem. Phys. 69, 1459-1461 (1978).

[47] M. Moskovits, Rev. Mod. Phys. 57, 783-826 (1985).

[48] A. Otto, in: Light Scattering in Solids IV. Electronic Scattering, Spin Effects, SERS and Morphic Effects, edited by M. Cardona, and G. Guntherodt (SpringerVerlag, Berlin, 1984), pp. 289-418.

[49] B. N. J. Persson, Chem. Phys. Lett. 82, 561-565 (1981).

[50] A. Campioni and P. Kambhampati, Chem. Soc. Rev. 27, 241-250 (1998).

[51] H. Guo et al., J. Phys. Chem. C 113, 10459-10464 (2009).

[52] S. J. Lee, Z. Guan, H. Xu, and M. Moskovits, J. Phys. Chem. C 111, 17985-17988 (2007).

[53] L. Rosa et al., J. Phys. Chem. C 115, 5251-5256 (2011).

[54] P. L. Stiles, J. A. Dieringer, N. C. Shah, and R. P. Van Duyne, Annu. Rev. Anal. Chem. 1, 601-626 (2008).

[55] Y. Xie, A. Zakharian, J. Moloney, and M. Mansuripur, Opt. Express 12, 6106-6121 (2004).

[56] W. L. Barnes, J. Opt. A. Pure Appl. Opt. 8, 87 (2006).

[57] Z. Liu et al., Nano Lett. 5, 1726-1729 (2005).

[58] W. Chen, D. C. Abeysinghe, R. L. Nelson, and Q. Zhan, Nano Lett. 9, 4320-4325 (2009)

[59] J. C. Love et al., Chem. Rev. 105, 1103-1169 (2005).

[60] Y. Fu et al., Opt. Express 18, 3438-3443 (2010).

[61] Z. Liu, J. M. Steele, H. Lee, and X. Zhang, Appl. Phys. Lett. 88, 171108 (2006).

[62] R. Mehfuz, M. W. Maqsood, and K. J. Chau, Opt. Express 18, 18206-18216 (2010).

[63] C. Genet and T. W. Ebbesen, Nature 445, 39-46 (2007). 